\title{
A shared-memory approach to microprocessor program development
}

\author{
HOWARD L. KAPLAN and DOMINIQUE DURAND \\ Addiction Research Foundation of Ontario, Toronto, Ontario M5S 2S1, Canada
}

\begin{abstract}
We have developed hardware and software interfaces with which part of a minicomputer's memory can be shared among its own programs and an Intel 8080 system. Although access to this shared memory is too slow for most real-time operations, the shared area is an excellent buffer for program code to be copied to faster memory for eventual real-time execution. The shared memory can also be used to pass test data between the processors to automate the testing of 8080 program modules.
\end{abstract}

There exist a number of commercially available microprocessor program development support systems. Such a system's hardware generally consists of a central processing unit, memory, mass storage peripherals, a terminal, perhaps a hard-copy printer, and a hardware interface to the microprocessor for which the software is to be developed. Except for the last interface, such a list resembles the configuration of many existing laboratory computer systems, and it is reasonable to ask what software and hardware need to be added to such a system to allow it to function for microprocessor program development support.

\section{CROSS-ASSEMBLY}

One readily available component of such a system is a cross-assembler, a program that will execute on one computer and produce object code that executes on a second computer. Unfortunately, many cross-assemblers are designed to replace the kind of assembler that executes on a minimal target microprocessor system. That is, in an attempt to be compatible with lowbudget peripherals, the user is encouraged to employ the kind of paper-tape technology on his microprocessor that should have been obsolete years ago, and produce output in paper-tape loader format. The user who is fortunate enough to have obtained a paper-tapeless configuration on some larger machine may find himself with no effective means to transfer assembled code from the larger to the smaller computer. Further refinements, such as producing code in a format suitable for the programming of PROMs, may also be missing from the design of the cross-assembler.

Last year, we obtained a cross-assembler. Our primary computer was then known as a Varian V76 and is now known as a Sperry-Univac V70-series computer, so we usually just call it the V76. The cross-assembler was obtained from the users' organization, VOICE, and conforms to the mnemonics and formats Intel had published for its own 8080 assembler. The crossassembler is written to execute under the control of the disk-based VORTEX II operating system. While it can produce standard paper-tape output, it also leaves the result of any assembly operations on a disk file in a straightforward format of two 8080 bytes to one 16-bit V76 word, located in proper positions on the 32K-word (64K-byte) disk file. We have written a number of support programs, and developed a hardware interface, that allow us to make effective use of this disk image in transferring programs from the V76 system to an 8080 development project.

\section{HARDWARE INTERFACE}

To briefly review 8080 architecture, the CPU accesses memory up to $64 \mathrm{~K} 8$-bit bytes, in addition to 256 I/O devices that are not normally memory mapped. However, the architecture will support addressing devices as memory, especially as there is a CPU input signal ("wait") that can suspend CPU operation indefinitely while a slow device reads or writes a word, acting as the CPU-specified address. Since stacks may be placed anywhere in memory and the interrupt reset vectors are normally located near the zero end, it is common to have a ROM monitor or initializer occupying the lowest memory locations. That is how the 8080 system was supplied to us.

Our 8080 system is an Intel SDK-80 kit, including an $8080 \mathrm{~A} \mathrm{CPU}$, a $1 \mathrm{~K}$ monitor in ROM, 256 bytes of RAM, a terminal interface, and locations in which to add more ROM, RAM, and additional components. The monitor handles inspection and modification of memory, copying of blocks of memory from one address to another, insertion and trapping of breakpoints, register modification, and terminal $\mathrm{I} / \mathrm{O}$. To this system we added an $\mathrm{I} / \mathrm{O}$ interface that would intercept all memory accesses from addresses 8000 to BFFF, the third quarter of memory, and send them along parallel $\mathrm{I} / \mathrm{O}$ cables to the V76, rather than to chips located on the 8080 board. Whenever such an access is requested, the interface logic puts the 8080 into an immediate wait state, with the memory addresses (and output data word, if 
any) held on the system busses. At the V76 end, a program eventually reads the 8080 address and data words as peripheral data, and uses them to access words in its own array memory. As soon as the data byte has been read from or sent to the interface, the 8080 waitstate signal is removed, allowing the CPU to continue processing. That is, from a functional standpoint, we are sharing memory between the two computers. From the standpoint of any 8080 software, these look like ordinary memory accesses, except that they are relatively slow. From the V76 standpoint, these are ordinary $\mathrm{I} / \mathrm{O}$ requests, not direct memory access requests, which allow the processing programs to provide intelligent intervention in the 8080 memory cycles.

With this interface constructed, it was necessary to make some slight changes in the on-board ROM monitor. Rather than using the I/O commands to communicate with a terminal, the monitor was modified to read from and write to memory address BFFF (the last location in shared memory) in order to receive and transmit terminal characters. In other words, we simply implemented some memory-mapped $\mathrm{I} / \mathrm{O}$ and let the $\mathrm{V} 76$ relay such requests back and forth to its own terminals. The VORTEX II system uses line-oriented, rather than character-oriented, software, so it is necessary to buffer and debuffer characters and lines; otherwise, the terminal handling is quite straightforward. To implement the change in the monitor, we read the contents of the original ROM into the V76 through a digital interface, used FORTRAN to convert the results to hexadecimal ASCII, wrote them to a disk file, entered patches in ASCII with a text editor, reread the results to convert them to binary, and programmed an Intel 2708-EPROM with the result.

\section{SOFTWARE INTERFACE}

At the V76 end of the interface, the processing program is known as $8080 \mathrm{DR}$, standing for 8080 driver. This program was written in FORTRAN, but uses assembler-coded subroutines to handle some of the special I/O functions and byte accessing. As this program must make a separate call or two to the I/O drivers for each data word transferred in either direction, and as such transfers involve the task-switching overhead of a multiprogramming system, our communications rate is fairly slow, about 300 address-data pairs per second. The 8080DR program contains an 8,192-word array, which is equivalent to the $16 \mathrm{~K}$ bytes of memory that the 8080 board maps through the computer-to-computer link. Whenever an address from 8000 to BFFF is transmitted over the interface, the $8080 \mathrm{DR}$ program checks additional status bits to determine whether it is a read or a write request and either fetches or stores an array byte (or terminal $\mathrm{I} / \mathrm{O}$ character) accordingly. The initial content of this array is a file produced by the crossassembler and its support programs; the content can consist either of code to be executed from the shared memory or of code to be copied to faster RAM chips for the testing of time-dependent routines. This is the most common use of the shared memory, as a temporary I/O buffer for RAM code, where the monitor's copying operation replaces reading assembled code from a peripheral $\mathrm{I} / \mathrm{O}$ device.

During the development of software, it is often useful to share the processing of data between the 8080 and the V76. For example, if the 8080 is logging data from its own peripherals into shared memory, it may be useful to have the V76 perform summary statistics on that data, until such time as the analysis can be performed on the 8080 . Also, it may be useful to have the V76 generate test data for 8080 routines, providing the original data and then inspecting the processed results. To accomplish these tasks, an auxiliary program runs in conjunction with $8080 \mathrm{DR}$. The program has access to the entire $16 \mathrm{~K}$ bytes of the shared memory. Whenever the 8080 requests that data be fetched from or stored into address BFFE, the auxiliary program executes before the memory cycle is allowed to complete. This allows the auxiliary program to place test data into memory, print summary statistics about data already in shared memory, or send special commands to alter the flow of the 8080 program. If we include an access to this location in an 8080 program test cycle, FORTRAN-coded auxiliary programs can conduct software tests with little human intervention. For example, we used this provision to test a multipleprecision binary-to-ASCII routine, having the auxiliary program generate each test value from 1 to 9,999 and store it in shared memory, one value per test. As the 8080 program converted each number to ASCII, it left the converted result in shared memory and then deposited an arbitrary value into location BFFE. At this point, the auxiliary program inspected the converted result, printed an error message if it was incorrect, and provided the 8080 with the next value to be converted. By this technique, we had a list of all improperly converted values and could look for a pattern (such as missed carry bits or numbers that crossed byte boundaries) among any errors generated.

In summary, the real-time operation is shared among three programs: one executing on the $8080 \mathrm{CPU}$ (though its memory accesses may all be mediated), one handling general memory and terminal requests on the V76, and one handling special test situations on the V76.

The mechanics of program assembly and storage are handled by five other programs in addition to the cross-assembler. The programs are organized on the assumption that the user will require no more than $16 \mathrm{~K}$ bytes of assembled code at any one time and that such code may be a mixture of material to be executed from shared memory and material to be copied to faster RAM or EPROM. The assembler handles the full $64 \mathrm{~K}$ byte address space of the 8080 , but we are careful to assemble our code as if the addresses were equivalent 
mod $16 \mathrm{~K}$. That is, if we are assembling code to be copied into RAM at 1000 to $13 \mathrm{FF}$, then we do not also generate code for 9000 to $93 \mathrm{FF}$ as part of the same assembly. By doing this, we fit all of the code for any one project into the $16 \mathrm{~K}$-byte shared-memory workspace.

Including the cross-assembler, the five programs are called ZEROSW, MCS80, CONDNS, 16KFIL, and PROMPR. ZEROSW simply clears the scratch disk file (named "SW") into which MCS80 assembles source files. After assembly, CONDNS scans that file for the first and last nonzero 16-bit word (pair of bytes) and stores only the code between those locations on a permanent disk file. The small programs are recombined by program $16 \mathrm{KFIL}$, which relocates them mod $16 \mathrm{~K}$ into a single $8 \mathrm{~K}$-word (16K-byte) array, reporting conflicts or overlaps as it loads each program. The resulting $16 \mathrm{~K}$-byte array can either be stored on disk as a single file or divided into $1 \mathrm{~K}$ files for use in programming 2708-type EPROMs by use of Program PROMPR.

\section{FUTURE POSSIBILITIES}

Our approach to microprocessor program development is conceptually similar to distributed processing. From the most obvious standpoint, any cross-assembly is a primitive form of distributed processing-one in which the two computers need share no physical data link. The shared-memory concept implements a more familiar form of distributed processing. Instead of remote file access, where a smaller computer can access records on other computers' disks as if they were on local disks, we have remote memory access, the same principle on the single-byte level. Finally, when during program testing we alternate processing of the shared array between the 8080 and the host V76 auxiliary program, we approach the level of tightly coupled CPUs.

Perhaps the most exciting prospect for distributing processing is to virtually eliminate the machineindependent parts of the on-board monitor. Some routines directly affect the microprocessor's state: fetch a byte into a register, store a byte, set a breakpoint and restore registers, trap a breakpoint and save registers. Most of the rest of the monitor simply oversees the order in which these operations occur. This suggests that a very simple monitor could be written, in which each of the primitive routines gets all of its control data, including the address of which primitive routine to execute next, from shared memory. All of the other logic, such as ASCII-to-binary conversion, command decoding, and testing for loop termination, would be performed in the host. It would even be possible to have no on-board monitor at all, only a virtual monitor consisting of reserved locations in shared memory. With such a simple monitor concept, it would require little work to develop the primitive subroutines and associated tables for an exotic microprocessor needed for a special application. In addition, the intelligence of the host minicomputer, monitoring all memory accesses, could be used to provide an instruction-by-instruction trace of all program execution, or even of selected parts of program execution, such as calls to particular subroutines or storage into selected memory locations.

We are interested in microprocessors as components of stand-alone biomedical measuring instruments. These are being developed for our own use, but we would like to design the units for possible sale to other users. Therefore, one of our concerns is to economize on memory and to avoid hardware multiplication and floating-point processing when simpler techniques suffice. For that reason, we are generally in the situation of needing to code our programs in assembler language, rather than in FORTRAN, BASIC, or another high-level language. However, there is no reason why a shared-memory interface cannot be used to transfer the object code from FORTRAN or PL/M to a microprocessor.

There is some concern that the microprocessors of the 1970s are simply bringing the software development shortcomings of the 1960 s to more users. In our view, the question of selecting a language to develop a program is not the only important query. At least as important is the quality of the support system, both hardware and software. Given the constraint that we need to write in assembler to get the quality of code required, we have a fast and comfortable system for developing programs for the 8080 . This approach should be quite suitable for adaptation to other microprocessors, host minicomputers, and laboratories. 